\title{
An Entropy-Based Model for Basal Ganglia Dysfunctions in Movement Disorders
}

\author{
Olivier Darbin, ${ }^{1,2}$ Daniel Dees, ${ }^{1}$ Anthony Martino, ${ }^{3}$ Elizabeth Adams, ${ }^{4}$ and Dean Naritoku ${ }^{1}$ \\ ${ }^{1}$ Department of Neurology, University of South Alabama, 307 University Boulevard, Mobile, AL 36608, USA \\ ${ }^{2}$ Division of System Neurophysiology, National Institute for Physiological Sciences, Okazaki, Aichi, Japan \\ ${ }^{3}$ Department of Neurosurgery, University of South Alabama, Mobile, AL 36608, USA \\ ${ }^{4}$ Department of Speech Pathology \& Audiology, University of South Alabama, Mobile, AL 36608, USA
}

Correspondence should be addressed to Olivier Darbin; odarbin@usouthal.edu

Received 27 February 2013; Accepted 6 May 2013

Academic Editor: Alessandro Sale

Copyright (c) 2013 Olivier Darbin et al. This is an open access article distributed under the Creative Commons Attribution License, which permits unrestricted use, distribution, and reproduction in any medium, provided the original work is properly cited.

\begin{abstract}
During this last decade, nonlinear analyses have been used to characterize the irregularity that exists in the neuronal data stream of the basal ganglia. In comparison to linear parameters for disparity (i.e., rate, standard deviation, and oscillatory activities), nonlinear analyses focus on complex patterns that are composed of groups of interspike intervals with matching lengths but not necessarily contiguous in the data stream. In light of recent animal and clinical studies, we present a review and commentary on the basal ganglia neuronal entropy in the context of movement disorders.
\end{abstract}

\section{Introduction}

Characterization of the neuronal data stream of basal ganglia neurons has been the foundation of most of the functional models for movement disorders. The divergences (or complementarities) between these models mostly result from the analytical strategy used to characterize and to model the data stream of the basal ganglia (BG) neurons. The analysis of the firing rate has forged the "rate hypothesis" while the frequency analysis has forged the "oscillatory model." Briefly, the rate hypothesis and oscillatory model suggest that increasing activity and/or beta oscillations in the output nuclei of the BG (the globus pallidus internal (GPi)) reduces motor selection and leads to hypokinesia in Parkinsonism.

Since this last decade, different groups have integrated new mathematical tools to characterize and/or model the activity of the BG neurons including nonlinear analyses which describe complex patterns in the neuronal data stream. After reviewing the recent findings from the nonlinear analysis of the BG neurons, we present a review on the avenues and hypotheses brought by these newly integrated mathematical tools and their possible impacts on the next generation of functional models of the basal ganglia.

\section{Linear Model for Basal Ganglia and Movement Disorders}

The basal ganglia are part of corticocortical loops (via the thalamus) [1-7] and have connections with the brainstem [8]. The striatum (Str) and subthalamic nucleus (STN) are the main input nuclei of the basal ganglia and receive excitatory glutaminergic input from the cortex. At the striatal level, this glutaminergic drive exerts a tonic excitatory control on the striatal efferent neurons (medium spiny neurons (MSN)) $[9,10]$ which project, via two pathways, to the basal ganglia output nuclei (the internal segment of the pallidum (GPi) and the pars reticulata of the substantia nigra $(\mathrm{SNr})$ ). The "direct" pathway is a GABAergic monosynaptic projection to these output nuclei, while the "indirect" pathway is a GABAergic multisynaptic projection via the globus pallidus external. An additional cortico-STN-GPi pathway provides a "hyperdirect" excitatory drive from STN to the GPi [11]. Therefore, neuronal activities in the basal ganglia output nuclei are dependent on the synergic activity between these pathways [12].

The sequential and convergent arrangement of excitatory and inhibitory neurons in these nuclei has forged the concept 
that basal ganglia and, inherently, information processing rely on the summation of excitatory and inhibitory inputs and are therefore linear in nature. To compare neuronal activity in the basal ganglia to the model predictions, the measurements of the firing rate and other linear markers in the time domain have been examined in animal and clinical studies. Data from these studies have contributed to the "rate model" for movement disorders. This model is founded upon the assumption that the direct pathway (Str-GPi/SNr) is up-regulated by the D1 dopaminergic receptor and indirect pathway (Str-GPe-GPi/SNr) is down-regulated by the D2 dopaminergic receptor. The dynamic balance between these two pathways contributes in motor selection and motor inhibition, respectively [13]. The "rate model" predicts that dopamine depletion leads to an imbalance in favor of the indirect pathway resulting in increased activity in GPi and excessive motor inhibition in Parkinsonism [14-22]. In dystonia, the model predicts a decreased activity in the GPi and increased selection of motor programs $[23,24]$. In contrast to a large body of experiments in animal models that favor the rate hypothesis, measurement of neuronal firing rates in the basal ganglia of patients with hypokinesia or hyperkinesia has given conflicting data with some studies supporting [25-31] and others not supporting the rate hypothesis [32-36].

In addition to time domain analyses which are based on the probability distribution of interspike intervals (ISIs), other studies have characterized the firing activity of basal ganglia neurons in the frequency domain. In a majority of studies, differences in oscillatory activities have been identified between normal and pathological conditions [3742]. In PD patients, oscillations in the beta range of $11-30 \mathrm{~Hz}$ have been reported to occur in approximately $30 \%$ of GPi neurons, while, in dystonia patients, lower beta frequencies in the $8-20 \mathrm{~Hz}$ are dominant but present in approximately $10 \%$ of GPi neurons [43].

\section{Evidence for Nonlinear Dynamic in the Neuronal Data Stream of BG Neurons}

The linear analyses used to characterize the basal ganglia activities in time and frequency domains measure the resultant linear combinations of independent patterns in the data stream. These analyses characterize the interspike interval (ISI) series by the summation of probability distributions for different durations of ISIs (i.e., firing rate, its range, or standard deviation) or several frequencies (power spectrum). However, the irregularity in the neuronal firing activity is not linear [44-51] since complex patterns can occur more often than predicted due to the probability distribution of the ISI series. In the last decade there has been a growing body of evidence that linear analysis does not fully describe the activity of neuronal networks and has justified the use of nonlinear analyses to further characterize the ISIs series [52-57]. These studies have identified neuronal patterns composed of either nonadjacent ISIs occurring nonperiodically, or patterns similar in shape but repeatedly occurring in different time scale (or size). The term "nonlinear temporal organization" has been introduced to define patterns identified by nonlinear analyses in the neuronal data stream. An initial finding by our group is that nonlinear temporal organization is present in a series of consecutive ISIs recorded from basal ganglia neurons in the awake normal primate [55] and in Parkinsonian patients [57]. These analyses have established that the temporal organization of ISIs in the time series results in the replication of complex patterns that cannot be statistically explained by random trials from the probability distribution of the ISIs [55-57].

\section{Sensitivity of Nonlinear Markers Regarding Pathological Conditions}

The clinical relevance of these nonlinear features in neuronal discharge is not yet clear. Specifically, it is unknown whether the nonlinear dynamics of basal ganglia neurons are affected by the conditions of parkinsonism or dystonia. In retrospective analyses of a database of PD and dystonia neurons with temporal organizations (as defined in [55]), Sanghera et al. [58] found a higher neuronal entropy (as estimated by the Approximate Entropy (ApEn) [59]) in the GPi of PD patients comparatively to dystonia patients. Currently, a major difficulty in the interpretation of these data, regarding the effects of the disorder per se, is the lack of comparison to the normal state of the basal ganglia. Nevertheless, these last data, in line with the decreases in neuronal entropy observed following deep brain stimulation (DBS) treatment in animal models for Parkinsonism [52] or apomorphine administration in PD patients [56], suggest that high neuronal entropy (at least in the GPi) is associated to lower kinetic activity. It is worth noting that, by construction, Approximate Entropy (ApEn) remains unchanged under uniform process magnification, reduction, and translation [59]. Therefore, changes in overall firing rate (per se) cannot explain the changes in neuronal entropy reported following treatments or between disorders.

Pharmacological studies in primate models for movement disorders are needed to further investigate this hypothesis as their basal ganglia neuronal activity exhibits similar patterns to those seen in patients [14]. In addition, it would be relevant to investigate whether similar markers can be identified in global signals, such as the EEG, which could provide a strategy to compare healthy condition to conditions with movement disorders in humans.

\section{The "Entropy Hypothesis"}

Since anti-Parkinsonian treatments decrease entropy and hyperkinetic conditions are associated with lower entropy, it is time to include the basal ganglia neuronal entropy as a putative interfering factor in the current model for the selection and the inhibition of motor information in the basal ganglia circuitry. Through exploring the current data framework available, we present a primary hypothesis on the nature of the GPi neuronal entropy regarding abnormal movement production.

From the data discussed above, we hypothesize that high entropy in the GPi neuronal data stream is associated to an increased motor inhibition (i.e., parkinsonism) while 


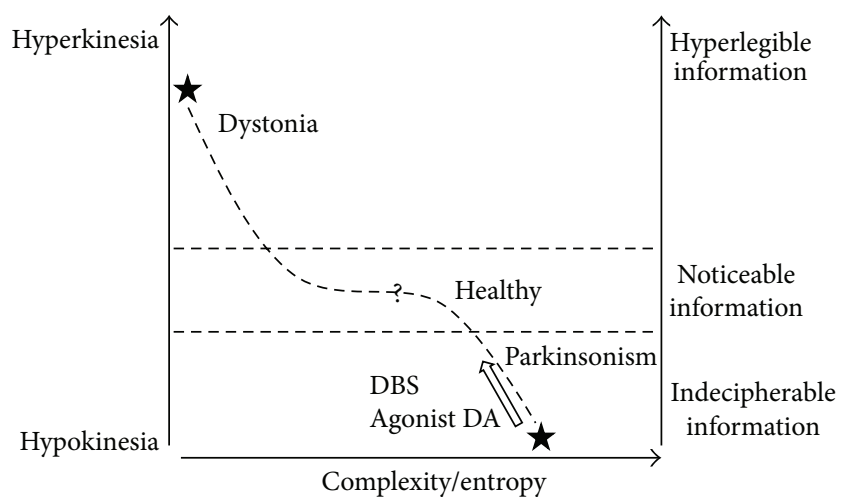

Figure 1: This figure shows a hypothetical relation between neuronal entropy and the aptitude of the downstream of information to generate movement regarding the conditions of hypokinesia and hyperkinesia. Low neuronal complexity could result in a hyperlegible signals and increased motor production. In contrast, high neuronal complexity could result in an indecipherable signal reducing motor production. Anti-Parkinsonian treatments reduce both hypokinesia and complexity. Since the comparisons between movement disorders and control remain unestablished, the healthy condition is envisaged with an adequate degree of GPi complexity allowing the emergence of noticeable information related to voluntary movement.

reduced entropy in the GPi neuronal data stream is envisaged as a feature for increasing motor selection (i.e., dystonia or anti-Parkinsonism treatments) (see Figure 1). This hypothesis underlies the idea that high entropy in the GPi neuronal data stream would correspond to a network condition generating a large number of different pattern possibilities leading to a signal with limited order or "organization" and reduced information. Therefore, the higher GPi neuronal entropy reported in parkinsonism can be conceptualized under Shannon-Brillouin's interpretation [60] as a measure of disorder, unpredictability, and reduced motor information leading to hypokinesia. This hypothesis is not opposed to the current model of selection and inhibition of motor programs along the basal ganglia circuitry [11] but introduces the GPi neuronal complexity as a factor which enhances the inhibition of motor program by decreasing the informative nature of the neuronal data stream. The "entropy hypothesis" predicts that lower entropy would increase information in the data stream and motor program selection resulting in hyperkinesia.

\section{Biological Substrata for the "Entropy Hypothesis"}

The relation between the entropy theory and the functions of the basal ganglia can be substantiated by the intrinsic (and logarithmic) relation between entropy and the correlation dimension [61]. Since the correlation dimension is a measure of the dimensionality of the space occupied by a data series, neuronal entropy can be envisaged as a related measurement of dimensionality of the neuronal data stream. The concept that correlated information from large population of neurons can be compressed into a selected number of neurons has been suggested to be an important mechanism for information encoding in the brain [62] and the basal ganglia especially [63]. High GPi neuronal entropy can underlie an inadequate compression (or reduction of the dimensionality [64]) of upstream population activity into the output neurons of the BG circuitry [63]. Alternatively, some circuitry reorganization such as increased interconnections between elements of the motor circuitry [65] may have the potential to increase the correlation dimension of the stream of information to the GPi neurons. Experimental research in animal models for movement disorders is warranted to explore these avenues.

\section{Conclusion}

The use of nonlinear domain analyses to describe the neuronal and network activities inside the basal ganglia may provide new qualitative and quantitative information regarding the nature of the sensory-motor processing as well as its distortion in pathological conditions. It is expected that the inclusion of key nonlinear features into siliconebased models of the basal ganglia could better reproduce complex and nonstationary signals recorded in normal and pathological conditions. To date, the "entropy hypothesis" may be useful to initiate a debate on nonlinear dynamics in basal ganglia activity and their roles in the selection and inhibition of motor programs. Most importantly, these nonlinear analyses may contribute to reduce the gap between the basal ganglia models and the theories on the processing of motor information.

\section{Acknowledgments}

Dr. OLivier Darbin is principally supported by the Department of Neurology University of South Alabama College of Medicine (Mobile, AL, USA) and by the Division of System Neurophysiology at the National Institute of Japan for Physiological Sciences ((NIJPS) Okazaki, Aichi, Japan).

\section{References}

[1] J. Yelnik, "Functional anatomy of the basal ganglia," Movement Disorders, vol. 17, supplement 3, pp. S15-S21, 2002.

[2] A. E. Pollack, "Anatomy, physiology, and pharmacology of the basal ganglia," Neurologic Clinics, vol. 19, no. 3, pp. 523-534, 2001.

[3] A. M. Graybiel, "The Basal ganglia," Current Biology, vol. 10, no. 14, pp. R509-R511, 2000.

[4] Y. Smith, M. D. Bevan, E. Shink, and J. P. Bolam, "Microcircuitry of the direct and indirect pathways of the basal ganglia," Neuroscience, vol. 86, no. 2, pp. 353-387, 1998.

[5] D. Joel and I. Weiner, "The organization of the basal gangliathalamocortical circuits: open interconnected rather than closed segregated," Neuroscience, vol. 63, no. 2, pp. 363-379, 1994.

[6] G. E. Alexander and M. D. Crutcher, "Functional architecture of basal ganglia circuits: neural substrates of parallel processing," Trends in Neurosciences, vol. 13, no. 7, pp. 266-271, 1990. 
[7] C. R. Gerfen, "The neostriatal mosaic. I. Compartmental organization of projections from the striatum to the substantia nigra in the rat," Journal of Comparative Neurology, vol. 236, no. 4, pp. 454-476, 1985.

[8] J. Mena-Segovia, J. P. Bolam, and P. J. Magill, "Pedunculopontine nucleus and basal ganglia: distant relatives or part of the same family?" Trends in Neurosciences, vol. 27, no. 10, pp. 585-588, 2004.

[9] A. R. West, S. B. Floresco, A. Charara, J. A. Rosenkranz, and A. A. Grace, "Electrophysiological interactions between striatal glutamatergic and dopaminergic systems," Annals of the New York Academy of Sciences, vol. 1003, pp. 53-74, 2003.

[10] O. Darbin and T. Wichmann, "Effects of striatal GABAAreceptor blockade on striatal and cortical activity in monkeys," Journal of Neurophysiology, vol. 99, no. 3, pp. 1294-1305, 2008.

[11] A. Nambu, "A new approach to understand the pathophysiology of Parkinson's disease," Journal of Neurology, vol. 252, no. 4, pp. ivl-iv4, 2005

[12] J. P. Bolam, J. J. Hanley, P. A. C. Booth, and M. D. Bevan, "Synaptic organisation of the basal ganglia," Journal of Anatomy, vol. 196, no. 4, pp. 527-542, 2000.

[13] C. R. Gerfen and D. J. Surmeier, "Modulation of striatal projection systems by dopamine," Annual Review of Neuroscience, vol. 34, pp. 441-466, 2011.

[14] A. Galvan and T. Wichmann, "Pathophysiology of Parkinsonism," Clinical Neurophysiology, vol. 119, no. 7, pp. 1459-1474, 2008.

[15] A. Nambu, "A new dynamic model of the cortico-basal ganglia loop," Progress in Brain Research, vol. 143, pp. 461-466, 2004.

[16] J. A. Obeso, C. Marin, C. Rodriguez-Oroz et al., "The basal ganglia in Parkinson's disease: current concepts and unexplained observations," Annals of Neurology, vol. 64, supplement 2, pp. S30-S46, 2008.

[17] J. K. H. Tang, E. Moro, N. Mahant et al., "Neuronal firing rates and patterns in the globus pallidus internus of patients with cervical dystonia differ from those with Parkinson's disease," Journal of Neurophysiology, vol. 98, no. 2, pp. 720-729, 2007.

[18] J. W. Mink, "The basal ganglia: focused selection and inhibition of competing motor programs," Progress in Neurobiology, vol. 50, no. 4, pp. 381-425, 1996.

[19] T. Boraud, E. Bezard, J. M. Stutzmann, B. Bioulac, and C. E. Gross, "Effects of riluzole on the electrophysiological activity of pallidal neurons in the 1-methyl-4-phenyl-1,2,3,6tetrahydropyridine-treated monkey," Neuroscience Letters, vol. 281, no. 2-3, pp. 75-78, 2000.

[20] A. Nambu, H. Tokuno, I. Hamada et al., "Excitatory conical inputs to pallidal neurons via the subthalamic nucleus in the monkey," Journal of Neurophysiology, vol. 84, no. 1, pp. 289-300, 2000.

[21] T. Wichmann and M. R. DeLong, "Functional neuroanatomy of the basal ganglia in Parkinson's disease," Advances in Neurology, vol. 91, pp. 9-18, 2003.

[22] M. R. DeLong and T. Wichmann, "Circuits and circuit disorders of the basal ganglia," Archives of Neurology, vol. 64, no. 1, pp. 20 24, 2007.

[23] D. Guehl, E. Cuny, I. Ghorayeb, T. Michelet, B. Bioulac, and P. Burbaud, "Primate models of dystonia," Progress in Neurobiology, vol. 87, no. 2, pp. 118-131, 2009.

[24] J. L. Vitek, "Pathophysiology of dystonia: a neuronal model," Movement Disorders, vol. 17, supplement 3, pp. S49-S62, 2002.
[25] L. E. Schrock, J. L. Ostrem, R. S. Turner, S. A. Shimamoto, and P. A. Starr, "The subthalamic nucleus in primary dystonia: singleunit discharge characteristics," Journal of Neurophysiology, vol. 102, no. 6, pp. 3740-3752, 2009.

[26] F. A. Lenz, J. I. Suarez, L. Verhagen Metman et al., "Pallidal activity during dystonia: somatosensory reorganisation and changes with seventy," Journal of Neurology Neurosurgery and Psychiatry, vol. 65, no. 5, pp. 767-770, 1998.

[27] M. Merello, D. Cerquetti, A. Cammarota et al., "Neuronal globus pallidus activity in patients with generalised dystonia," Movement Disorders, vol. 19, no. 5, pp. 548-554, 2004.

[28] M. K. Sanghera, R. G. Grossman, C. G. Kalhorn et al., "Basal ganglia neuronal discharge in primary and secondary dystonia in patients undergoing pallidotomy," Neurosurgery, vol. 52, no. 6, pp. 1358-1373, 2003.

[29] P. A. Starr, G. M. Rau, V. Davis et al., "Spontaneous pallidal neuronal activity in human dystonia: comparison with Parkinson's disease and normal macaque," Journal of Neurophysiology, vol. 93, no. 6, pp. 3165-3176, 2005.

[30] "General discussion of drug therapy in dystonia," Advances in Neurology, vol. 14, pp. 417-422, 1976.

[31] J. I. Lee, L. Verhagen Metman, S. Ohara, P. M. Dougherty, J. H. Kim, and F. A. Lenz, "Internal pallidal neuronal activity during mild drug-related dyskinesias in Parkinson's disease: decreased firing rates and altered firing patterns," Journal of Neurophysiology, vol. 97, no. 4, pp. 2627-2641, 2007.

[32] H. C. Walker, R. L. Watts, C. J. Schrandt et al., "Activation of subthalamic neurons by contralateral subthalamic deep brain stimulation in parkinson disease," Journal of Neurophysiology, vol. 105, no. 3, pp. 1112-1121, 2011.

[33] J. K. H. Tang, E. Moro, A. M. Lozano et al., "Firing rates of pallidal neurons are similar in Huntington's and Parkinson's disease patients," Experimental Brain Research, vol. 166, no. 2, pp. 230-236, 2005.

[34] A. Stefani, A. Bassi, P. Mazzone et al., "Subdyskinetic apomorphine responses in globus pallidus and subthalamus of parkinsonian patients: lack of clear evidence for the 'indirect pathway,"' Clinical Neurophysiology, vol. 113, no. 1, pp. 91-100, 2002.

[35] R. Levy, J. O. Dostrovsky, A. E. Lang, E. Sime, W. D. Hutchison, and A. M. Lozano, "Effects of apomorphine on subthalamic nucleus and globus pallidus internus neurons in patients with Parkinson's disease," Journal of Neurophysiology, vol. 86, no. 1, pp. 249-260, 2001.

[36] E. B. Montgomery, "Subthalamic nucleus neuronal activity in Parkinson's disease and epilepsy subjects," Parkinsonism and Related Disorders, vol. 14, no. 2, pp. 120-125, 2008.

[37] M. D. Bevan, P. J. Magill, D. Terman, J. P. Bolam, and C. J. Wilson, "Move to the rhythm: oscillations in the subthalamic nucleus-external globus pallidus network," Trends in Neurosciences, vol. 25, no. 10, pp. 525-531, 2002.

[38] P. Brown, "Abnormal oscillatory synchronisation in the motor system leads to impaired movement," Current Opinion in Neurobiology, vol. 17, no. 6, pp. 656-664, 2007.

[39] C. Hammond, H. Bergman, and P. Brown, "Pathological synchronization in Parkinson's disease: networks, models and treatments," Trends in Neurosciences, vol. 30, no. 7, pp. 357-364, 2007.

[40] A. Eusebio and P. Brown, "Synchronisation in the beta frequency-band-the bad boy of parkinsonism or an innocent bystander?" Experimental Neurology, vol. 217, no. 1, pp. 1-3, 2009. 
[41] P. Gatev, O. Darbin, and T. Wichmann, "Oscillations in the basal ganglia under normal conditions and in movement disorders," Movement Disorders, vol. 21, no. 10, pp. 1566-1577, 2006.

[42] M. Weinberger and J. O. Dostrovsky, "A basis for the pathological oscillations in basal ganglia: the crucial role of dopamine," NeuroReport, vol. 22, no. 4, pp. 151-156, 2011.

[43] M. Weinberger, W. D. Hutchison, M. Alavi et al., "Oscillatory activity in the globus pallidus internus: comparison between Parkinson's disease and dystonia," Clinical Neurophysiology, vol. 123, no. 2, pp. 358-368, 2011.

[44] H. Korn and P. Faure, "Is there chaos in the brain? II. Experimental evidence and related models," Comptes Rendus, vol. 326, no. 9, pp. 787-840, 2003.

[45] G. J. Mpitsos, R. M. Burton, H. C. Creech, and S. O. Soinila, "Evidence for chaos in spike trains of neurons that generate rhythmic motor patterns," Brain Research Bulletin, vol. 21, no. 3, pp. 529-538, 1988.

[46] M. A. Mendez, P. Zuluaga, R. Hornero et al., "Complexity analysis of spontaneous brain activity: effects of depression and antidepressant treatment," Journal of Psychopharmacology, vol. 26, no. 5, pp. 636-643, 2011.

[47] A. Friedman, I. Deri, Y. Friedman et al., "Decoding of dopaminergic mesolimbic activity and depressive behavior," Journal of Molecular Neuroscience, vol. 32, no. 1, pp. 72-79, 2007.

[48] R. A. Silver, "Neuronal arithmetic," Nature Reviews Neuroscience, vol. 11, no. 7, pp. 474-489, 2010.

[49] M. London and M. Häusser, "Dendritic computation," Annual Review of Neuroscience, vol. 28, pp. 503-532, 2005.

[50] A. Bülent UşaklI, "Modeling of movement-related potentials using a fractal approach," Journal of Computational Neuroscience, vol. 28, no. 3, pp. 595-603, 2010.

[51] R. Kozma, S. Bressler, L. Perlovsky, and G. K. Venayagamoorthy, "Advances in neural networks research: an introduction," Neural Networks, vol. 22, no. 5-6, pp. 489-490, 2009.

[52] A. D. Dorval, G. S. Russo, T. Hashimoto, W. Xu, W. M. Grill, and J. L. Vitek, "Deep brain stimulation reduces neuronal entropy in the MPTP-primate model of Parkinson's disease," Journal of Neurophysiology, vol. 100, no. 5, pp. 2807-2818, 2008.

[53] W. Li, D. Jia, J. L. Wang et al., "Deterministic dynamics in neuronal discharge from pallidotomy targets," Journal of International Medical Research, vol. 36, no. 5, pp. 979-985, 2008.

[54] M. Rodríguez, E. Pereda, J. González, P. Abdala, and J. A. Obeso, "Neuronal activity in the substantia nigra in the anaesthetized rat has fractal characteristics. Evidence for firing-code patterns in the basal ganglia," Experimental Brain Research, vol. 151, no. 2, pp. 167-172, 2003.

[55] O. Darbin, J. Soares, and T. Wichmann, "Nonlinear analysis of discharge patterns in monkey basal ganglia," Brain Research, vol. 1118, no. 1, pp. 84-93, 2006.

[56] M. Lafreniere-Roula, O. Darbin, W. D. Hutchison, T. Wichmann, A. M. Lozano, and J. O. Dostrovsky, "Apomorphine reduces subthalamic neuronal entropy in parkinsonian patients," Experimental Neurology, vol. 225, no. 2, pp. 455-458, 2010.

[57] J. Lim, M. K. Sanghera, O. Darbin, R. M. Stewart, J. Jankovic, and R. Simpson, "Nonlinear temporal organization of neuronal discharge in the basal ganglia of Parkinson's disease patients," Experimental Neurology, vol. 224, no. 2, pp. 542-544, 2010.

[58] M. K. Sanghera, O. Darbin, M. Alam et al., "Entropy measurements in pallidal neurons in dystonia and Parkinson's disease," Movement Disorders, vol. 27, supplement 1, pp. S1-639, 2012.
[59] S. Pincus, "Approximate entropy (ApEn) as a complexity measure," Chaos, vol. 5, no. 1, pp. 110-117, 1995.

[60] G. Longo, P. A. Miquel, C. Sonnenschein, and A. M. Soto, "Is information a proper observable for biological organization?" Progress in Biophysics and Molecular Biology, vol. 109, no. 3, pp. 108-114, 2012.

[61] S. Zurek, P. Guzikb, S. Pawlakc, M. Kosmidera, and J. Piskorski, "On the relation between correlationdimension, approximateentropy and sample entropy parameters, and a fast algorithm for their calculation," Physica A, vol. 391, pp. 6601-6610, 2012.

[62] H. B. Barlow, "Single cells versus neuronal assemblies," in Information Processing in the Cortex: Experiments and Theory, pp. 169-174, Springer, 1992.

[63] D. S. Andres, D. Cerquetti, and M. Merello, "Finite dimensional structure of the GPI discharge in patients with parkinson's disease," International Journal of Neural Systems, vol. 21, no. 3, pp. 175-186, 2011.

[64] I. Bar-Gad, G. Morris, and H. Bergman, "Information processing, dimensionality reduction and reinforcement learning in the basal ganglia," Progress in Neurobiology, vol. 71, no. 6, pp. 439-473, 2003.

[65] R. M. Villalba and Y. Smith, "Differential structural plasticity of corticostriatal and thalamostriatal axo-spinous synapses in MPTP-treated parkinsonian monkeys," Journal of Comparative Neurology, vol. 519, no. 5, pp. 989-1005, 2011. 

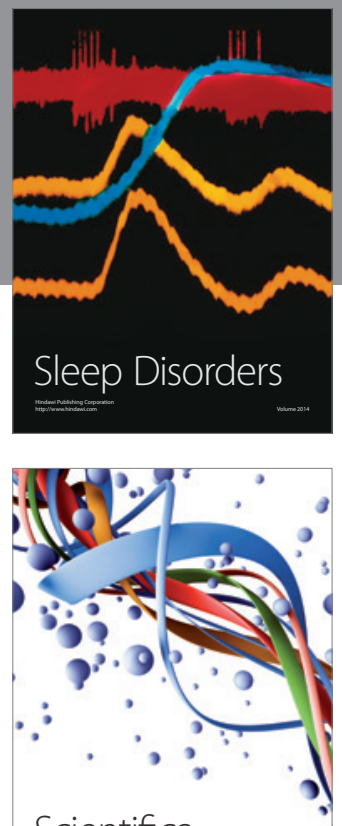

Scientifica
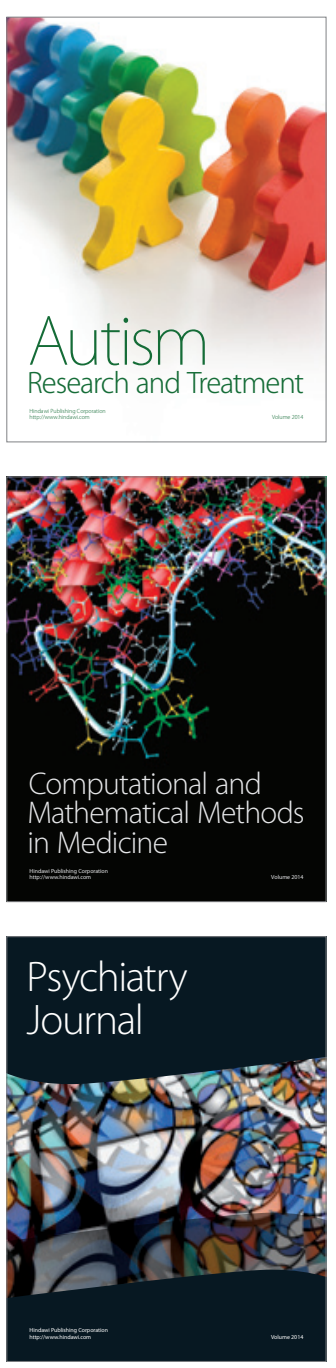
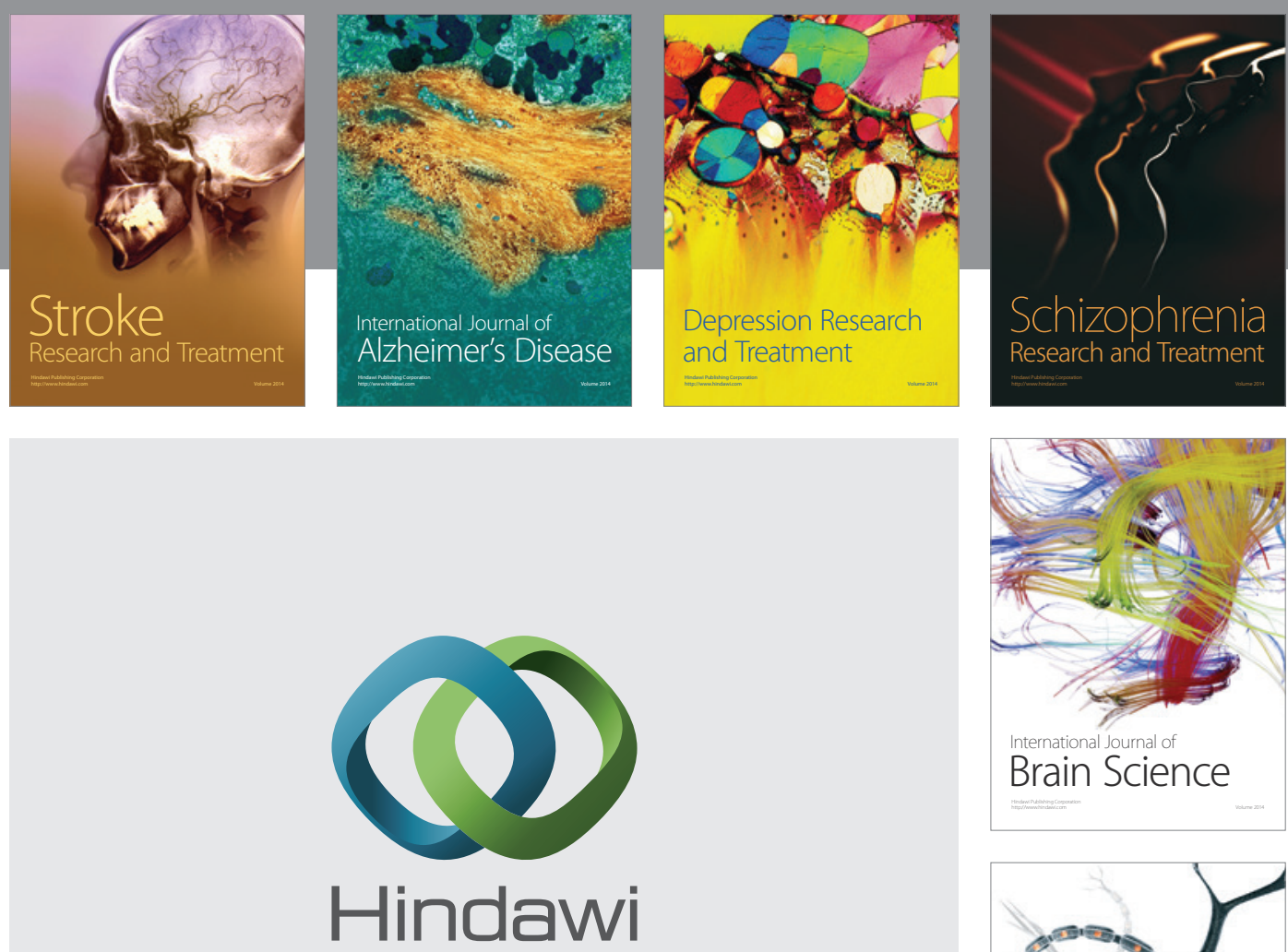

Submit your manuscripts at

http://www.hindawi.com
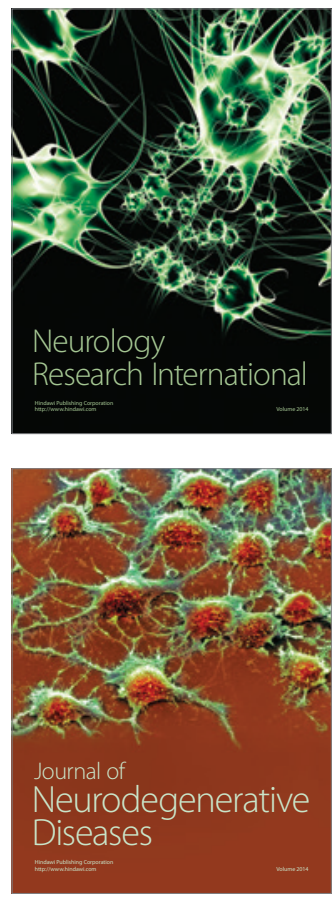

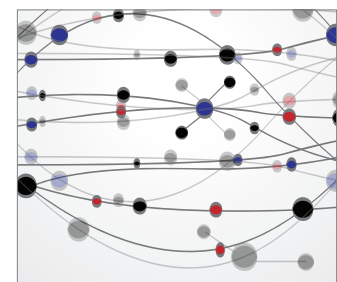

The Scientific World Journal
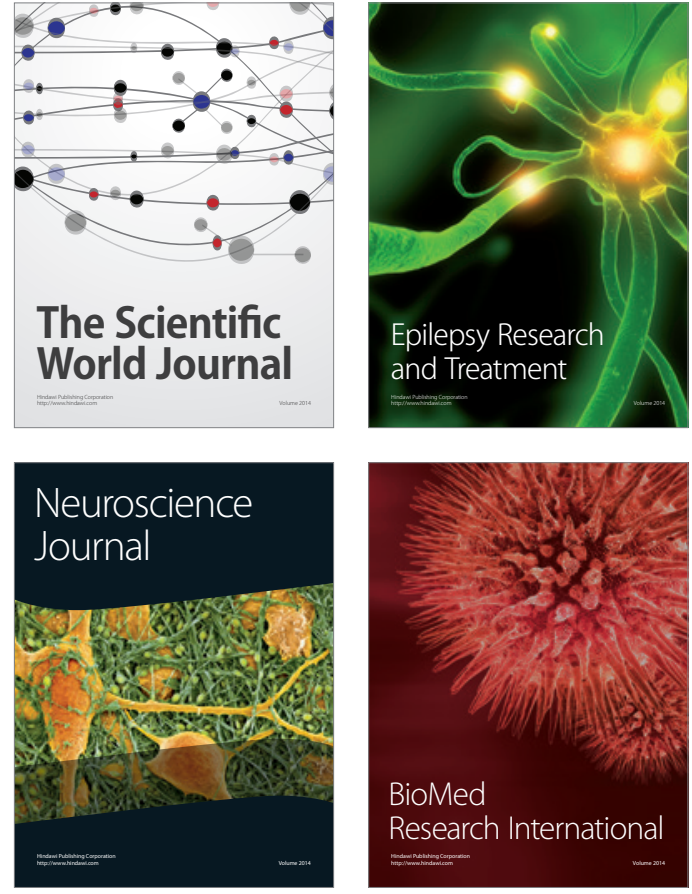

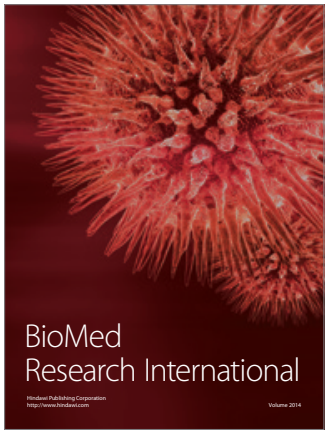

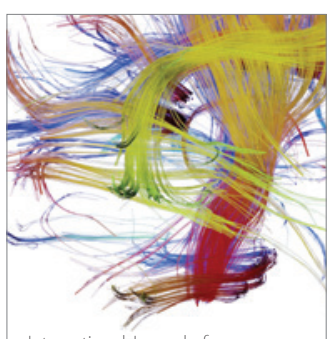

Brain Science

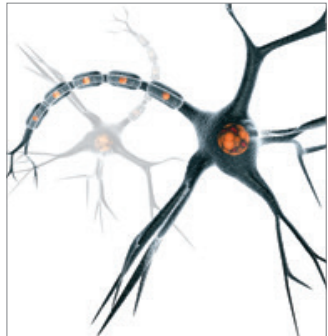

Neural Plasticity
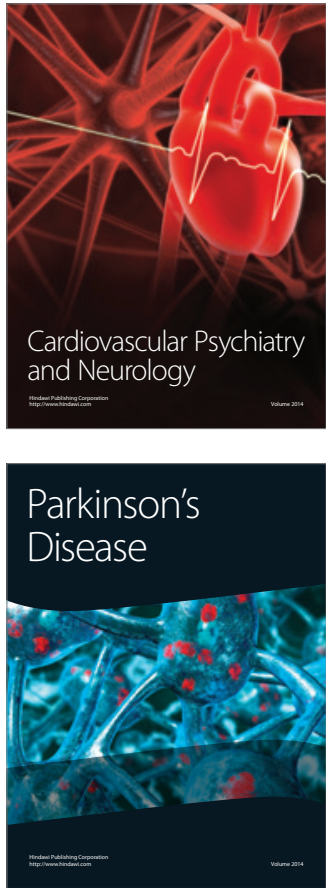